welche man als $\gamma$-Bibrompropionsäure bezeichnen könnte, und welche mit nascirendem Wasserstoff in Propionsäure übergehen wird.

XIV. Ueber die $\alpha$-Monobromacrylsäure und Ueberführung der $\alpha$-Bibrompropionsäure in $\beta$-Säure; von Denselben.

Darstellung der $\alpha$-Monobromacrylsäure.

Die Analogie der beiden Bibrompropionsäuren mit Propylenbromür und Methylbromacetol, von denen sie sich nur durch Ersetzung von $\mathrm{CH}^{3}$ durch $\mathrm{COOH}$ unterscheiden, hat uns dazu geführt, eine der mit den beiden letzteren Isomeren ausgeführten Reactionen, nämlich die Reaction gegen Aetzkali, wobei $\mathrm{HBr}$ austritt, auch an der $\alpha$-Bibrompropionsäure $\mathrm{zu}$ versuchen, besonders da $\mathrm{Ri} \mathrm{ch}$. W a gner und der Eine ${ }^{*}$ ) von uns mit der $\beta$-Säure auf diese Weise zu bestimmten Resultaten gekommen sind (siehe folgende Abhandlung), indem sich Bromkalium bildet und monobromacrylsaures Kali bald in schönen Blättchen absetzt.

Die ersten Versuche zeigten uns schon, dafs die Reaction ziemlich schwierig von Statten geht, denn beim Kochen einer Lösung von $\alpha$-Bibrompropionsäure in Alkohol mit Kali dauert es geraume Zeit, bis sich Bromkalium abzuscheiden beginnt, und vergeblich warteten wir auf das Erscheinen der beim Arbeiten mit der $\beta$-Säure beobachteten schönen Blättchen.

*) Diese Annalen 16z, 232 Anmerkung; Berichte der deutschen chemischen Gesellschaft B, 512. 
334 Philippiu. Tollens, üb.d. $\alpha$-Monobromacrylsäure

In diesen Versuchen haben wir folgende Mengen $\alpha$-Säure und absoluten Alkohol angewandt :

$\begin{array}{cccccc}20 & \text { Grm. Säure } & \text { und } & 100 & \text { Alkohol } \\ 20 & n & n & n & 40 & n \\ 15 & n & \eta & n & 50 & n \\ 13 & n & n & n & 40 & n \\ 15 & \eta & n & n & 60 & n\end{array}$

In dem Alkohol hatten wir vorher die nach der Gleichung : ${ }_{a} \mathrm{C}^{3} \mathrm{H}^{4} \mathrm{Br}^{2} \mathrm{O}^{2}+2 \mathrm{KOH}=\mathrm{KBr}+\mathrm{C}^{3} \mathrm{H}^{2} \mathrm{Br}^{2} \mathrm{O}^{2} \mathrm{~K}+2 \mathrm{H}^{2} \mathrm{O}$ $a$-Bibrompropionsäure monobromacrylsaures Kali

berechnete Menge käufliches Aetzkali von bekanntem Gehalt gelöst und ihn vom Bodensatze decantirt, nachher kochten wir das Gemenge längere Zeit am Rückflufskühler.

Es schieden sich meist nur unbedeutende Mengen Bromkalium ab, die sich beim Erkalten vermehrten, bis sich zuletzt wenig andere Krystalle beizumengen anfingen, worauf die Lösung abgegossen und auf flachen Schalen verdampft wurde.

Zu unserem Erstaunen waren jedoch die so erhaltenen Krystalle meist nicht das gewünschte Salz, sondern einfach $\alpha$-bibrompropionsaures Kali, welches trotz des langen Kochens der Zerstörung widerstanden hatte und sich durch mehrere der auf S. 320 angeführten Analysen, sowie durch die Eigenschaften der wieder in Freiheit gesetzten und durch Ausschütteln mit Aether gewonnenen Säure als solches documentirte.

Einmal jedoch erhielten wir das gewünschte monobromacrylsaure Kali, doch in sehr geringer, nur zu einer Kaliumbestimmung ausreichenden Menge (Nr. III, S. 335).

Bessere Ausbeute erhielten wir bei Anwendung von 3 Mol. Kalihydrat auf 1 Mol. $\alpha$-Bibrompropionsäure, und drei mit je $10 \mathrm{Grm}$. $\alpha$-Säure *) ausgeführte Operationen gaben uns genügende Mengen Monobromacrylsäure.

*) Da die $\beta$-Säure ebenfalls monobromacrylsaures Kali hefert und zwar viel leichter (siehe S. 341), verwandten wir besondere Sorg- 
wnd Veberführung d. $\alpha$-Bibrompropionsäure in $\beta$-Säure. 335

Das nach mehrstündigem Kochen, wie oben beschrieben, durch Verdunsten der vom Bromkalium abgegossenen alkoholischen Lösung erhaltene Salz wurde mehrfach aus Wasser umkrystallisirt und abgeprefst, bis eine Probe sich durch fehlende Reaction mit verdünnter Silberlösung als frei von Bromkalium erwies.

Das bei langsamem Verdunsten erhaltene monobromacrylsaure Kali bildet sehr schöne rhombische Tafeln, welche der $\boldsymbol{\beta}$-Bibrompropionsäure ähneln, und sowohl beim Krystallisiren gröfserer Mengen, als auch beim Verdunsten eines Tröpfchens unter dem Mikroscop gut zu beobachten sind.

I. 0,2421 Grm. gaben 0,1707 $\mathrm{CO}^{2}$ und $0,0364 \mathrm{H}^{2} \mathrm{O}$.

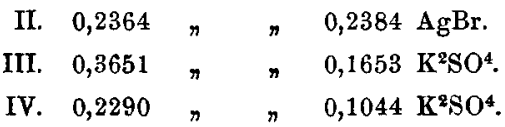

\begin{tabular}{lccccc} 
& & \multicolumn{4}{c}{ Gefunden } \\
$\mathrm{C}^{8}$ & $\mathbf{1 9 , 0 4}$ & I. & II. & III. & IV. \\
$\mathrm{H}^{2}$ & 1,06 & $1,63,23$ & - & - & - \\
$\mathrm{Br}$ & 42,31 & - & - & - & - \\
$\mathrm{O}^{2}$ & 16,92 & - & - & - & - \\
$\mathrm{K}$ & 20,67 & - & - & 20,32 & $20,47$.
\end{tabular}

Die freie Monobromacrylsäure erhielten wir aus dem Kaliumsalze durch Lösen desselben in wenig Wasser, Versetzen mit Schwefelsäure und Ausschütteln mit Aether (siehe folgende Abhandlung, S. 343), welcher sie als krystallinische, in Wasser und Alkohol leicht lösliche, die Haut stark angreifende Masse hinterliefs. Sie bildet nach wiederholtem

falt auf die Reinigung der zu diesen Versuchen dienenden Säure, um dem Vorwurfe zu begegner, es sei vielleicht eine geringe Menge einer anderen Bibronipropionsäure darin enthalten und Ursache der Bildung der Monobromacrylsäure gewesen. Nur genau bei 60 bis $61^{\circ}$ schmelzende völlig weifse Säure kam zur Anwendung. 
Schmelzen mit einer Spur Wasser, Erstarren und Abpressen schöne, besonders unter dem Mikroscop gut zu beobachtende rechtwinkelige Tafeln, welche constant bei 69 bis $70^{\circ}$ schmelzen.

$0,2138 \mathrm{Grm}$. gaben $0,1895 \mathrm{CO}^{2}$ und $0,049 \mathrm{H}^{2} \mathrm{O}$.

$\begin{array}{ccc} & \text { Berechnet } & \text { Gefunden } \\ \mathrm{C}^{3} & 23,84 & 24,17 \\ \mathrm{H}^{3} & 1,99 & 2,55 \\ \mathrm{Br} & 52,98 & - \\ \mathrm{O}^{2} & 21,19 & -\end{array}$

Die Säure kann nicht lange aufbewahrt werden, denn sie stöfst nach einiger Zeit Bromwasserstoff aus und wandelt sich in eine weiche bräunliche Masse um.

Letzterer Umstand, wie Schmelzpunkt und Krystallform, nähern diese Monobromacrylsäure sehr der anderen, aus $\boldsymbol{\beta}$-Bibrompropionsäure erhaltenen, von $\mathrm{W}$ agner und dem Einen von uns untersuchten (siehe folgende Abhandlung), so dafs wir versucht waren, beide für identisch zu halten; die Eigenschaften der Kaliumsalze differenziren sie jedoch völlig, indem das unserige stets rhombische Tafeln, das Wagn er'sche dagegen grolse rechtwinkelige Blätter bildet, welche Formen bei allen Versuchen, sie durch Hinzufügung von Spuren der isomeren Substanzen zu beeinflussen, immer constant blieben.

Wir unterscheiden demnach unsere aus $\alpha$-Bibrompropionsäure erhaltene Säure als $\alpha$-Monobromacrylsäure von der isomeren, von Wagner und Tollens als $\not \dot{\beta}$ bezeichneten.

Ihre Constitution und Bildung wird durch folgende Formeln ausgedrückt :

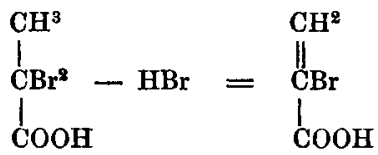

$\boldsymbol{\alpha}$-Bibrompropionsäure $\alpha$-Bromacrylsäure. 
und Ueberführung d. $\alpha$-Bibrompropionsäure in $\boldsymbol{\beta}$-Säure. 337 Bildung der $\beta$-Bibrompropionsäure aus $\alpha$-Monobromacrylsäure.

Interessant war der Versuch, unserer Säure wieder $\mathbf{H B r}$ zuzufiihren, da auf diese Weise die Bildung sowohl von $\alpha-$ als auch von $\boldsymbol{\beta}$-Bibrompropionsäure denkbar ist.

$\mathrm{Zu}$ diesem Zwecke erhitzten wir etwas ganz reine a-Monobromacrylsäure mit ihrem 3- bis 4 fachen Gewicht rauchender Bromwasserstoffsäure einige Stunden im zugeschmolzenen Rohr auf $100^{\circ}$, dampften darauf die etwas gefärbte Säure auf ein geringes Volum ab und liefsen erkalten, worauf sich besonders beim Berühren mit einem Körnchen $\boldsymbol{\beta}$-Bibrompropionsäure die schönen rhombischen Täfelchen dieser Säure abschieden, während im Gegentheil ein Berühren der im Krystallisiren begriffenen Säure mit $\alpha$-Bibrompropionsäure Zerfliefsen bewirkte.

Es ergiebt sich aus diesem mehrfach immer mit demselben Resultat angestellten Versuche die merkwiirdige Thatsache, dafs sich aus der aus a-Bibrompropionslure entstandenen a-Monobromacrylslure nicht wieder dieselbe $a$-, sondern die isomere $\beta$-Bibrompropionsäure bildet. Es hat sich also durch diese Operationen eine isomere Säure aus der anderen gebildet.

Directe Umwandlung von $\alpha$-Bibrompropionsäure in $\beta$-Säure.

Nach Ausfiihrung der bescbriebenen Versuche kam uns der Gedanke, das Verhalten der a-Bibrompropionsaure gegen rauchende Bromwasserstoffslure zu priifen, indem während des Erhitzens abwechselndes Abspalten und Wiederanlagern von $\mathrm{HBr}$ dissociationsartig stattinden konnte.

Hierzu erhitzten wir $6 \mathrm{Grm}$. reine a-Bibrompropionsiiure mit $4 \mathrm{Grm}$. rauchendem $\mathrm{HBr}$ im zugeschmolzenen Rohr auf 


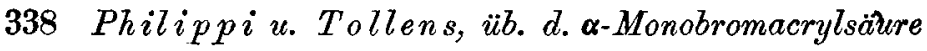
$100^{\circ}$, indem wir täglich offneten *) und von Zeit zu Zeit Proben nahmen. Zuerst schied sich die in der Wärme stets gelöste Bibrompropionsäure in Krystallen aus, später als Oel, endlich blieb sie gelöst.

Nach achttägigem Erhitzen dampften wir den Röhrennhalt im Wasserbade $a b$, worauf sich nach dem Erkalten über Schwefelsäure die wohlbekannten Rhomben der $\beta$-Bibrompropionsäure zeigten; sie wurden durch mehrfaches $A b-$ pressen und Umkrystallisiren gereinigt und besafsen den von Münder und Tollens beobachteten Schmelzpunkt $64^{\circ}$.

Wir könnten diese directe Umwandlung von $\alpha$-Bibrompropionsäure in $\beta$-Säure als exquisites Beispiel einer molecularen Umlagerung, hervorgerufen durch Wärme und den Contact mit rauchender Bromwasserstoffsäure, anführen; doch ziehen wir vor, dies unvollkommene Erklärung für die Fälle zu reserviren, in welchen es keine bessere giebt, und glauben, dafs die besprochene Umwandlung einfach durch allmäliges abwechselndes Abspalten und Wiederanlagern von $\mathrm{HBr}$ zu Stande gekommen ist, und gründen diefs auf das Verhalten der $\alpha$-Bibrompropionsäure beim Erhitzen, wobei sie sich unter Abgabe von $\mathrm{HBr}$ theilweise zersetzt; hierbei wird sie Monobromacrylsäure geben, welche bei Gegenwart von überschüssigem $\mathrm{HBr}$ mit diesem sich wieder verbindet :

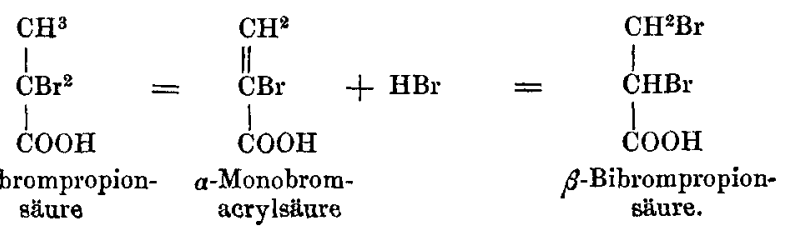

Die bei $220^{\circ}$ ziemlich lebhafte Zersetzung der $\alpha$-Bibrompropionsäure wird bei $100^{\circ}$ und bei Gegenwart des überschüssigen $\mathrm{HBr}$ langsam vor sich gehen und deshalb ein acht

*) Es zeigte sich meist gelinder Druck im Inneren der Röhren. 
Tage lang dauerndes Erhitzen erforderlich. Es läfst sich dieses Zerfallen und Wiederbilden mit manchen Dissociationsvorgängen vergleichen, bei denen ehenfalls die Molecule sich successive trennen und vereinigen; der Unterschied ist nur, dafs die getrennten Molecule sich nicht auf dieselbe Weise wieder vereinigen, wie diefs vorher der Fall gewesen war.

Die Entstehung von $\beta$-Bibrompropionsäure aus $\alpha$-Säure *) ist von Wichtigkeit, weil sie eine neue Bestltigung der von uns vertheidigten Structur der Acrylsäure bildet. In der aus Propionsäure hergestellten a - Bibrompropionsäure ist die Existenz der Carboxylgruppe über jeden Zweifel erhaben, indem im entgegengesetzten Falle wohl kaum irgend eine Formel mehr sicherstehen würde. Der Austritt und die Wiederanlagerung von $\mathrm{HBr}$ lassen sich ungezwungen nur erkilären, wenn man die Carboxylgruppe hierbei intact läfst; denn eine anderweitig vielleicht nach Art des Epichlorhydrins ***) $\mathrm{zu}$ construirende Zwischenverbindung würde jedenfalls keine so entschiedene, der Monochloressigsäure an die Seite zu stellende Säure sein, wie es die Monobromacrylsäure ist.

*) Ein Versuch, aus $\beta$-Bibrompropionsăure vielleicht durch Erhitzen auf hohe Temperatur umgekehrt zur $\alpha$-Säure au gelangen, schlug fehl. Wir erhitzten $10 \mathrm{Grm}$. $\beta$-Säure im eugeschmolzenen Rohr vier Wochen lang auf $230^{\circ}$ und erhielten, nachdem wir häufig den Inhalt der Röhre geprüft and immer unter den Zersetzungsprodncten noch Rhomben von $\beta$-Säure erkannt hatten, zuletzt eine braune, schmierige Flüssigkeit, die sich der weiteren Untersuchung unzugänglich zeigte. Eben so wenig Erfolg hatte ein Versuch, $\beta$-Säare durch langes Erhitzen mit rauchendem $\mathrm{HBr}$ auf $230^{\circ}$ in $a$-Säure umzuwandeln. Dit Säure löste sich auf, schied sioh erst als Krystalle, dann als Oel, endlich gar nicht mehr ab, und beim Abdampfen der Lösung blieb eị Syrup, der nicht zum Krystallisiren gu bringen war.

$\mathrm{CE}^{3}$

*) Etwa $\stackrel{\stackrel{\mathrm{CBr}}{!}}{\mathrm{CO}}\}$ O ? 
Somit ist also die Carboxylgruppe in der $\beta$-Bibrompropionsäure und ferner in der daraus entstehenden Acrylsäure enthalten.

\section{Ueber die $\beta$-Monobromacrylsäure aus} $\beta$-Bibrompropionsäure ;

von Rich. Wagner und B. Tollens.

Die vorliegende Abhandlung ist zum Theil von dem Wunsche veranlafst worden, von $\operatorname{der} \beta$-Bibrompropionsäure einen Uebergang zur $\alpha$-Säure zu finden, um die Gleichheit der Structur beider Säuren zu beweisen. $\mathrm{Zu}$ diesem $\mathrm{Zwecke}$ suchten wir aus der $\beta$-Säure ein Molecul Bromwasserstoff abzuspalten, Bromacrylsäure zu bilden und den Bromwasserstoff nachher wieder anzulagern, und hofften auf diese Weise nicht wieder $\beta$ - sondern $\alpha$-Bibrompropionsäure zu erhalten. In dieser letzten Hinsicht haben wir nur theilweisen Erfolg gehabt; wir haben aus $\beta$-Bibrompropionsäure die Monobromacrylsäure erhalten, aber gefunden, dafs sie beim Wiederanlagern von Bromwasserstoff von neuem die $\beta$-Säure liefert, und diefs Resultat steht im Einklange mit den aus der vorigen Abhandlung sich ergebenden, denn bei mäfsig hoher Temperatur scheinen die die Bibrompropionsäure bildenden Atome überhaupt Neigung zu haben, die Lage der $\beta$-Säure anzunehmen. Die Monobromacrylsäure haben wir einer genauen Untersuchung unterworfen, sie als $\beta$-Säure von der von Philippi und Tollens hergestellten $\alpha$-Bromacrylsäure unterschieden, ihre Constitution festgestellt und einige nicht unwichtige Zersetzungsproducte derselben studirt (siehe folgende Abhandlung XVI.). 